\title{
Accuracy of Two Circulating Antigen Tests for the Diagnosis and Surveillance of Schistosoma mansoni Infection in Low-Endemicity Settings of Côte d'Ivoire
}

\author{
Rufin K. Assaré, ${ }^{1,2,3,4 *}$ Mathieu I. Tra-Bi, ${ }^{1}$ Jean T. Coulibaly, ${ }^{1,2,3,4}$ Paul L. A. M. Corstjens, ${ }^{5}$ Mamadou Ouattara, ${ }^{1,2}$ \\ Eveline Hürlimann, ${ }^{3,4}$ Govert J. van Dam, ${ }^{6}$ Jürg Utzinger, ${ }^{3,4}$ and Eliézer K. N'Goran ${ }^{1,2}$ \\ ${ }^{1}$ Unité de Formation et de Recherche Biosciences, Université Félix Houphouët-Boigny, Abidjan, Côte d'Ivoire; ${ }^{2}$ Centre Suisse de Recherches \\ Scientifiques en Côte d'Ivoire, Abidjan, Côte d'Ivoire; ${ }^{3}$ Swiss Tropical and Public Health Institute, Basel, Switzerland; ${ }^{4}$ University of Basel, Basel, \\ Switzerland; ${ }^{5}$ Department of Cell and Chemical Biology, Leiden University Medical Center, Leiden, The Netherlands; ${ }^{6}$ Department of Parasitology, \\ Leiden University Medical Center, Leiden, The Netherlands
}

\begin{abstract}
In low-endemicity settings, current tools for the diagnosis and surveillance of schistosomiasis are often inaccurate in detecting true infection. We assessed the accuracy of an up-converting phosphor lateral flow circulating anodic antigen (UCP-LF CAA) test and a point-of-care circulating cathodic antigen (POC-CCA) urine cassette test for the diagnosis of Schistosoma mansoni. Our study was conducted in eight schools of western Côte d'Ivoire. Fifty children, aged 9-12 years, were enrolled per school. From each child, a single urine specimen and two stool specimens were collected over consecutive days for diagnostic work-up. Urine samples were subjected to UCP-LF CAA and POC-CCA tests. From each stool sample, triplicate Kato-Katz thick smears were examined. Overall, 378 children had complete data records. The prevalence of S. mansoni, as assessed by six Kato-Katz thick smears, was 4.0\%. The UCP-LF CAA and POC-CCA tests revealed S. mansoni prevalence of $25.4 \%$ and $30.7 \%$, respectively, when considering trace results as positive, and prevalence of $23.3 \%$ and $10.9 \%$ when considering trace results as negative. In the latter case, based on a composite "gold" standard, the sensitivity of UCP-LF CAA (80.7\%) was considerably higher than that of POC-CCA (37.6\%) and six Kato-Katz thick smears (13.8\%). The negative predictive value of UCP-LF CAA, POC-CCA, and six Kato-Katz thick smears was $92.8 \%$, $79.8 \%$, and $74.1 \%$, respectively. Our results confirm that UCP-LF CAA is more accurate than Kato-Katz and POC-CCA for the diagnosis of $S$. mansoni in low-endemicity settings.
\end{abstract}

\section{INTRODUCTION}

Schistosomiasis remains a public health problem in many parts of sub-Saharan Africa. ${ }^{1,2}$ The global burden of schistosomiasis is estimated at 1.4 million disability-adjusted life years (DALYs). ${ }^{3}$ The life cycle of Schistosoma requires a human as definitive host, specific freshwater snails as intermediate host, and human-water contacts. Adult Schistosoma worms live in the gastrointestinal or urinary tract, where they produce eggs, which pass through stool or urine. Schistosomiasis can lead to anemia, malnutrition, and stunted growth in children, and bladder cancer in adults. ${ }^{4}$

There are several approaches for schistosomiasis control, including snail control; water, sanitation, and hygiene; information, education, and communication; and, most importantly, preventive chemotherapy. ${ }^{1}$ The latter strategy consists of mass drug administration (MDA) with praziquantel, targeting at-risk groups (e.g., school-age children) without prior diagnosis. In 2016, 68.5 million school-age children from 30 African countries received praziquantel. ${ }^{5}$ The implementation of preventive chemotherapy significantly reduced the prevalence of schistosomiasis in many parts of Africa. ${ }^{6,7}$ In settings where the endemicity of schistosomiasis has been lowered due to preventive chemotherapy, efforts should be made to further reduce the prevalence of heavy-intensity infections to levels $<1 \%$ (as determined by egg counts), to eliminate schistosomiasis as a public health problem. ${ }^{8}$ Rigorous monitoring and surveillance in such settings is required so that pockets of transmission can be rapidly identified and tailored public health responses implemented.

Accurate diagnosis is an important prerequisite for monitoring and surveillance of schistosomiasis. With regard to

*Address correspondence to Rufin K. Assaré, Unité de Formation et de Recherche Biosciences, Université Félix Houphouët-Boigny, 22 BP 582 Abidjan 22, Côte d'Ivoire. E-mail: hrufinass@yahoo.fr
Schistosoma mansoni, the Kato-Katz technique is the most widely used approach in epidemiologic surveys assessing the impact of preventive chemotherapy. However, in lowendemicity settings, the Kato-Katz technique lacks sensitivity. Examination of multiple Kato-Katz thick smears, ideally obtained from consecutive stool samples, enhances the sensitivity of the Kato-Katz technique. ${ }^{9,10}$ However, collection of multiple stool samples is time-consuming and costly and reduces compliance in parasitologic survey. Serological methods, such as ELISA and indirect hemagglutination test, and molecular techniques, such as polymerase chain reaction (PCR) and loop-mediated isothermal amplification are highly sensitive. ${ }^{11-13}$ Yet, in most schistosomiasis-endemic countries, these diagnostic tests are not available. Hence, there is a need for diagnostic tools that have high sensitivity and specificity and can be available at the point-of-care (POC), particularly in settings where the prevalence and intensity of infection are low.

Prior research has shown that the POC circulating cathodic antigen (CCA) urine cassette test and the up-converting phosphor lateral flow circulating anodic antigen (UCP-LF CAA) assay are more sensitive than the standard Kato-Katz technique for S. mansoni diagnosis. ${ }^{14-18}$ UCP-LF CAA assay has a particularly high sensitivity and specificity, and hence, might be especially well suited for detection of low infection intensities. ${ }^{19}$ Although CAA can be detected both in urine and serum, a urine UCP-LF CAA test can be more sensitive than a serum UCP-LF CAA test due to the adaptability to use large sample volume. ${ }^{20-22}$ Collection of urine rather than blood usually results in higher compliance.

We report on a study conducted in the western part of Côte d'Ivoire. We purposefully enrolled eight schools where the prevalence of $S$. mansoni based on the Kato-Katz technique, after several rounds of MDA with praziquantel, was very low. ${ }^{15}$ Our objective was to determine the accuracy of UCP-LF CAA, 
POC-CCA, and multiple Kato-Katz thick smears for the diagnosis of S. mansoni.

\section{METHODS}

Ethics statements. The study was approved by the Comité National d'Ethique et de la Recherche of the Ministry of Health in Côte d'Ivoire (reference no. 046/MSHP/CNER-kp; date of assignment: May 30, 2016). Before the parasitologic survey, health and education authorities and village leaders received information about the objectives and procedures of the study. Potential risks and benefits were explained to the communities in lay terms. Parents or legal guardians signed a written informed consent, and children provided oral assent. Participation was voluntary, and children could withdraw at any time without further obligation. At the end of the study, all school-age children in the study villages were offered praziquantel free of charge, according to guidelines put forward by the World Health Organization (WHO). ${ }^{23}$

Study area and population surveyed. Details of the study area and population surveyed have been described elsewhere. $^{15}$ In brief, we designed a cross-sectional study in 14 schools in three regions in the western part of Côte d'Ivoire, extending from $06^{\circ} 32^{\prime} 42.0^{\prime \prime}$ to $07^{\circ} 36^{\prime} 54.8^{\prime \prime} \mathrm{N}$ latitude and from $06^{\circ} 44^{\prime} 09.8^{\prime \prime}$ to $07^{\circ} 33^{\prime} 48.9^{\prime \prime}$ W longitude. The S. mansoni prevalence among 9- to 12-year-old children ranged from nil to $22 \%$, as determined by the Kato-Katz technique, and the corresponding values varied from nil to $33 \%$, as assessed by POC-CCA urine cassette test if trace results were considered positive. Eight schools with a low S. mansoni prevalence ( $<10 \%$ according to Kato-Katz) were selected to assess the accuracy of POC-CCA and UCP-LF CAA compared with multiple Kato-Katz thick smears.

Urine and stool collection. In each of the eight schools, 50 children aged 9-12 years who had a written informed consent form signed by parents or guardians, were enrolled. The first day of the survey, children received two prelabeled plastic containers and were invited to return the containers filled with a fresh morning stool and a urine sample. Upon collection, children received another prelabeled plastic container and were asked to provide a second stool sample the next day. Stool and urine samples were transferred to a nearby laboratory at the Hôpital Général de Douékoué or the Centre Hospitalier Regional de Man.

Stool and urine examination. In the laboratory, stool samples were subjected to triplicate $41.7 \mathrm{mg}$ Kato-Katz thick smears. ${ }^{24}$ Each child provided two stool samples, and in total, six Kato-Katz thick smears per child were examined; the average of the numbers of eggs was used in the analysis. After a clearing time of $30 \mathrm{~min}$, the Kato-Katz thick smears were examined under a microscope by one of four experienced laboratory technicians. Eggs of S. mansoni, as well as Ascaris lumbricoides, Enterobius vermicularis, hookworm, Hymenolepis spp., Taenia spp., and Trichuris trichiura, were counted and recorded for each species separately. Here, only the results pertaining to $S$. mansoni are presented. The cumulative number of $S$. mansoni eggs on the six Kato-Katz thick smears (total volume approximately $250 \mathrm{mg}$ of stool) was multiplied by a factor of 4 to obtain an estimate of infection intensity, proxied by the number of $S$. mansoni eggs per $1 \mathrm{~g}$ of stool (EPG). Infection intensity of S. mansoni was stratified into three categories: 1) light (1-99 EPG), 2) moderate
(100-399 EPG), and 3) heavy ( $\geq 400$ EPG) according to guidelines put forward by WHO. ${ }^{25}$

Urine samples were tested using the POC-CCA assay (Rapid Medical Diagnostics; Pretoria, South Africa; batch number: 50182). ${ }^{26}$ In brief, one drop of urine was added to the well of the cassette test. Next, one drop of test buffer was added, according to the manufacturer's protocol (note that the currently offered POC-CCA test format is adapted for use without buffer). The cassette test was read after 20 min by an experienced laboratory technician and results were categorized as negative, trace, or positive.

S. haematobium eggs were identified and quantified using urine filtration method. In addition, an aliquot of $5 \mathrm{~mL}$ of urine was frozen. The aliquots were transferred to a laboratory at the Centre Suisse de Recherches Scientifiques en Côte d'Ivoire (Abidjan, Côte d'Ivoire) and stored at $-20^{\circ} \mathrm{C}$. Frozen urine samples were shipped to the Department of Parasitology, Leiden University Medical Center (LUMC; Leiden, The Netherlands) and kept at $-20^{\circ} \mathrm{C}$ pending further analysis.

At LUMC, urine samples were subjected to UCP-LF CAA. The UCP-LF CAA test was performed using $2 \mathrm{~mL}$ of urine (UCAA2000, wet assay format), which allows detecting 0.1 $\mathrm{pg} / \mathrm{mL}$ CAA. ${ }^{27,28}$ The results of the UCAA2000 assay were stratified according to two approaches. For the first approach (fine-grained classification), the results were stratified into six categories as follow: 1) urine sample is considered high positive at CAA levels of $>100 \mathrm{pg} / \mathrm{mL}, 2$ ) positive at 10 to $100 \mathrm{pg} /$ $\mathrm{mL}, 3$ ) low positive at 1 to $10 \mathrm{pg} / \mathrm{mL}$, 4) very low positive at 0.1 to $1 \mathrm{pg} / \mathrm{mL}, 5$ ) indecisive at 0.05 to $0.1 \mathrm{pg} / \mathrm{mL}$, and 6) negative at CAA levels of $<0.05 \mathrm{pg} / \mathrm{mL}$. In the second approach (coarser classification), the UCAA2000 results were stratified into three categories: 1) urine sample considered positive at CAA levels of $>0.1 \mathrm{pg} / \mathrm{mL}, 2$ ) indecisive at 0.05 to $0.1 \mathrm{pg} /$ $\mathrm{mL}$, and 3) as negative at $<0.05 \mathrm{pg} / \mathrm{mL}$. Of note, $50 \%$ of indecisive results may be true positives when tested with larger sample volume. ${ }^{21} \mathrm{~A}$ sample returning a CAA level in the indecisive range in this study may be indicated as a trace result for conformity with the POC-CCA terminology.

Statistical analysis. Data were entered into Microsoft Excel 2010 (Microsoft Corporation, Redmond, WA) and analyzed with STATA version 15.1 (Stata Corporation, College Station, TX). In the absence of a diagnostic "gold" standard, we defined a composite "gold" standard, as described elsewhere. $^{29}$ In short, a child was considered positive when at least one $S$. mansoni egg was found on any of the six Kato-Katz thick smears, and/or a positive POC-CCA, and/or a positive UCP-LF CAA test result was obtained. POC-CCA trace and UCP-LF CAA indecisive results were considered as negative. On the basis of this approach, sensitivity and negative predictive value (NPV) of the diagnostic tools were determined. The nonparametric Spearman's rank correlation test was applied to measure the correlation between CAA $\mathrm{pg} / \mathrm{mL}$ levels and the fecal egg count (expressed in EPG) or POC-CCA scores (negative, trace, and positive).

The strength of agreement between the diagnostic tests was assessed by Kappa statistics $(\kappa)$. In brief $\kappa=\frac{p o-p e}{1-p e}$, where $p o$ is the observed proportion of agreement and $p e$ is the proportion of agreement expected by chance. $\kappa=0$ indicates no agreement; $\kappa=0.0$ to 0.2 indicates poor agreement; $\kappa=0.21$ to 0.4 indicates fair agreement; $\kappa=0.41$ to 0.6 indicates moderate agreement; $\kappa=0.61$ to 0.8 indicates substantial agreement; and $\kappa=0.81$ to 1.0 indicates almost perfect 


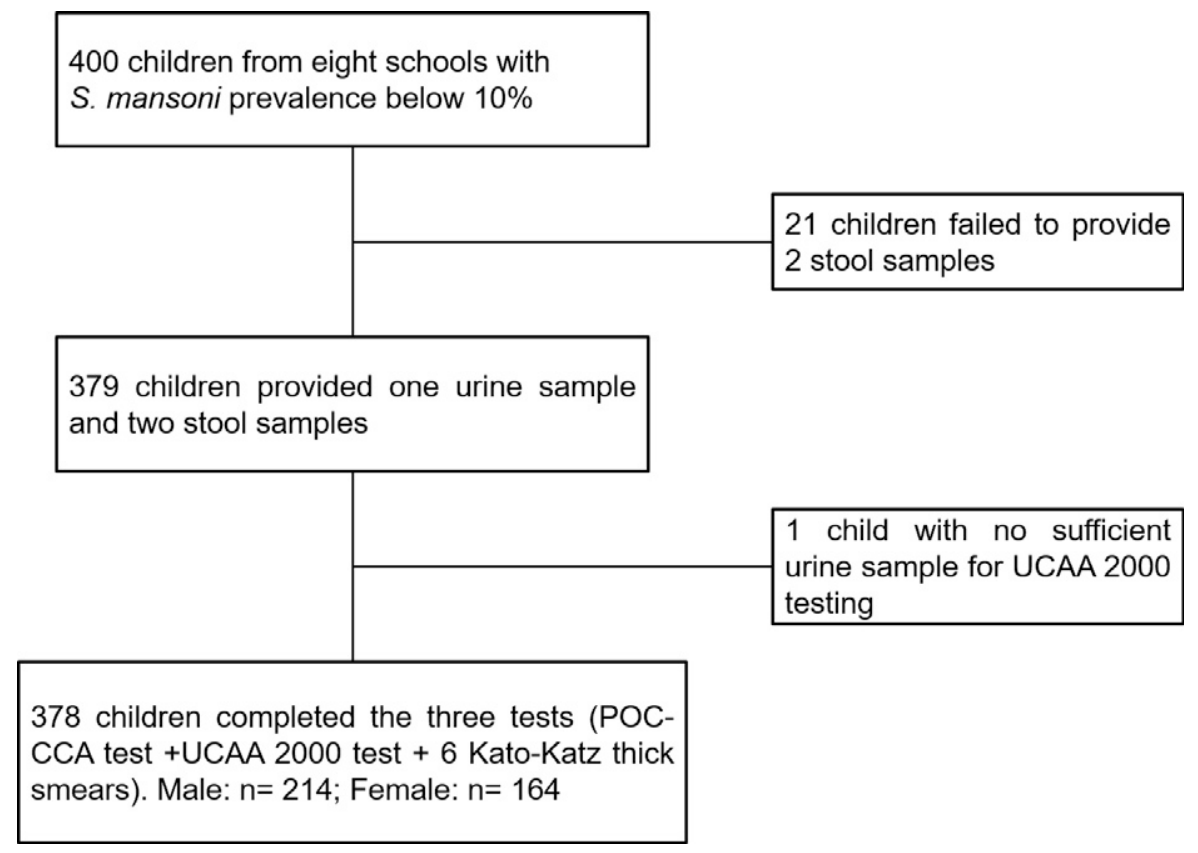

FIGURE 1. Flow chart detailing study participation.

agreement. $^{30}$ The same degrees of agreement were applied for the Spearman rank correlation.

\section{RESULTS}

Population characteristics. A total of 400 children, aged 9-12 years from eight schools, had informed consent signed by their parents or guardians, and hence, they were invited to participate. Twenty-one children failed to provide two stool samples over consecutive days, and one child had an insufficient amount of urine for the UCP-LF CAA test. Taken together, 378 children had complete data records (i.e., two stool samples, each subjected to triplicate Kato-Katz thick smears, and one urine sample subjected to both POC-CCA and UCP-LF CAA) (Figure 1). The mean age of the 378 children was 10.4 years with considerably more boys than girls (214 versus 164).

S. mansoni prevalence according to different diagnostic techniques. Table 1 summarizes the prevalence of $S$. mansoni, stratified by school (village) and the diagnostic test used. Stool examination with six Kato-Katz thick smears revealed a $S$. mansoni prevalence of $4.0 \%$ (15/378) with a range at the unit of the school from nil (Gregbeu) to $6.3 \%$ (Semien). The intensity of S. mansoni infection, expressed as arithmetic mean among $S$. mansoni egg-positive children, was 49.1 EPG (95\% confidence interval [CI]: 20.0-78.1 EPG). The lowest mean intensity of infection amongst eggpositive children was 4.0 EPG (Siambly), and the highest value was 88.0 EPG (Mahinahi). Two children with the highest infection level would fall in the moderate infection intensity (100 and 192 EPG) according to WHO definition.

On the basis of UCP-LF CAA test, there were $74.6 \%, 2.1 \%$, $10.6 \%, 5.3 \%, 2.7 \%$, and $4.8 \%$ of negative, indecisive, very low positive, low positive, positive, and high positive results, respectively. Considering a coarser classification, there were $74.6 \%, 2.1 \%$, and $23.3 \%$ negative, indecisive, and positive results. When considering indecisive (trace) results as positive, the prevalence of $S$. mansoni was $25.4 \%$ (96/378), ranging between $12.0 \%$ (Siambly) and $62.0 \%$ (Zouatta II). Considering indecisive results as negative, the S. mansoni

TABLE 1

Prevalences of Schistosoma mansoni among children aged 9-12 years in eight villages of western Côte d'Ivoire, stratified by diagnostic approaches

\begin{tabular}{|c|c|c|c|c|c|c|c|c|c|c|c|}
\hline \multirow[b]{2}{*}{ School } & \multirow[b]{2}{*}{$\begin{array}{l}\text { No. of children } \\
\text { examined }\end{array}$} & \multicolumn{2}{|c|}{$\begin{array}{l}\text { Sextuplicate } \\
\text { Kato-Katz }\end{array}$} & \multicolumn{2}{|c|}{$\begin{array}{c}\text { UCP-LF CAA } \\
\text { Indecisive positive }\end{array}$} & \multicolumn{2}{|c|}{$\begin{array}{c}\text { UCP-LF CAA } \\
\text { Indecisive negative }\end{array}$} & \multicolumn{2}{|c|}{$\begin{array}{l}\text { POC-CCA } \\
\text { Trace positive }\end{array}$} & \multicolumn{2}{|c|}{$\begin{array}{c}\text { POC-CCA } \\
\text { Trace negative }\end{array}$} \\
\hline & & $\begin{array}{l}\text { No. } \\
\text { positive }\end{array}$ & $\%$ & $\begin{array}{l}\text { No. } \\
\text { positive }\end{array}$ & $\%$ & $\begin{array}{l}\text { No. } \\
\text { positive }\end{array}$ & $\%$ & $\begin{array}{l}\text { No. } \\
\text { positive }\end{array}$ & $\%$ & $\begin{array}{l}\text { No. } \\
\text { positive }\end{array}$ & $\%$ \\
\hline Gregbeu & 47 & 0 & 0.0 & 7 & 14.9 & 6 & 12.8 & 11 & 23.4 & 11 & 23.4 \\
\hline Klangbolably & 50 & 3 & 6.0 & 9 & 18.0 & 9 & 18.0 & 16 & 32.0 & 4 & 8.0 \\
\hline Mahinahi & 36 & 1 & 2.8 & 10 & 27.8 & 9 & 25.0 & 15 & 41.6 & 7 & 19.4 \\
\hline Mona & 47 & 2 & 4.3 & 11 & 23.4 & 11 & 23.4 & 17 & 36.2 & 6 & 12.8 \\
\hline Semien & 48 & 3 & 6.3 & 11 & 22.9 & 9 & 18.8 & 10 & 20.8 & 4 & 8.3 \\
\hline Siambly & 50 & 1 & 2.0 & 6 & 12.0 & 4 & 8.0 & 12 & 24.0 & 1 & 2.0 \\
\hline Zouatta II & 50 & 3 & 6.0 & 31 & 62.0 & 30 & 60.0 & 22 & 44.0 & 5 & 10.0 \\
\hline Zê & 50 & 2 & 4.0 & 11 & 22.0 & 10 & 20.0 & 13 & 26.0 & 3 & 6.0 \\
\hline Total & 378 & 15 & 4.0 & 96 & 25.4 & 88 & 23.3 & 116 & 30.7 & 41 & 10.9 \\
\hline
\end{tabular}


TABLE 2

Sensitivity and NPV of different tools for diagnosis of Schistosoma mansoni

\begin{tabular}{lcc}
\hline \multicolumn{1}{c}{ Diagnostic approach* } & Sensitivity & $\mathrm{NPV}$ \\
\hline Kato-Katz $(6 \times)$ & 13.8 & 74.1 \\
95\% Cl & $7.9-21.7$ & $69.3-78.5$ \\
UCP-LF CAA† & 80.7 & 92.8 \\
95\% Cl & $72.1-87.7$ & $89.1-95.5$ \\
POC-CCA & 37.6 & 79.8 \\
95\% Cl & $28.5-47.4$ & $75.1-84.0$ \\
UCP-LF CAA + Kato-Katz (6×) & 83.5 & 93.7 \\
95\% Cl & $75.2-89.9$ & $90.3-96.2$ \\
UCP-LF CAA + Kato-Katz (6×) & 42.2 & 81.0 \\
95\% Cl & $32.8-52.0$ & $76.4-85.1$ \\
UCAA2000 + POC-CCA & 97.2 & 98.9 \\
95\% Cl & $92.2-99.4$ & $96.8-99.8$ \\
\hline
\end{tabular}

CAA = circulating anodic antigen; CCA = circulating cathodic antigen; $\mathrm{Cl}=$ confidence interval; NPV = negative predictive value; POC = point-of-care; UCP-LF = up-converting phosphor lateral flow.

* Gold standard: Participant considered positive if he was found S. mansoni egg positive and/ or found UCP-LF CAA test positive and/or POC-CCA test positive.

† Considered UCP-LF CAA indecisive as negative results.

$\ddagger$ Considered POC-CCA trace negative results.

prevalence was slightly lower (23.3\%), ranging from $8.0 \%$ (Siambly) to $60.0 \%$ (Zouatta II). The UCP-LF CAA test (UCAA2000 format) revealed a more than 5-fold higher $S$. mansoni prevalence compared with six Kato-Katz thick smears.

Considering trace results as positive, there were 116 $(30.7 \%)$ children with a positive POC-CCA urine cassette test result. At the unit of the school, the prevalence ranged from $20.8 \%$ (Semien) to $44.0 \%$ (Zouatta II). The overall prevalence decreased to $10.9 \%$ when POC-CCA trace results were counted as negative with the lowest prevalence of $2.0 \%$ observed in Siambly and the highest prevalence of $23.4 \%$ estimated for Gregbeu.

On the basis of a single urine filtration, $S$. haematobium eggs were observed in only two children ( $0.5 \%$ overall), including one child in Zê (2\%) and one child in Zouatta II (2\%). In Zê, the urine filtration-positive child was also positive by KatoKatz, POC-CCA, and UCP-LF CAA. In Zouatta II, the S. haematobium egg-positive child was also positive by Kato-Katz and UCP-LF CAA, but negative by POC-CCA.

Diagnostic accuracy. Table 2 shows the accuracy of the individual diagnostic assays when considering the composite test as diagnostic "gold" standard. We determined a sensitivity of $80.7 \%, 37.6 \%$, and $13.8 \%$ for UCP-LF CAA, POC-CCA, and six Kato-Katz thick smears, respectively. The combination of UCP-LF CAA and POC-CCA together had the highest sensitivity (97.2\%). The NPV was $92.8 \%, 79.8 \%$, and $74.1 \%$ for UCP-LF CAA, POC-CCA, and six Kato-Katz thick smears, respectively. The combination of UCP-LF CAA and POC-CCA revealed higher NPV (98.9\%) than UCP-LF CAA and KatoKatz (93.7\%) or POC-CCA and Kato-Katz (81.0\%).

Correlation and agreement between diagnostic assays. There was a fair, yet significant positive relationship between urine CAA levels $(\mathrm{pg} / \mathrm{mL})$ and fecal $S$. mansoni egg counts (Spearman's rho $=0.29 ; P<0.001$ ), between CAA concentrations and POC-CCA score (Spearman's rho $=0.35 ; P<$ 0.001 ), and between $S$. mansoni egg counts and POC-CCA score (Spearman's rho $=0.32 ; P<0.001$ ) (Figure 2).

Table 3 summarizes the number of positive and negative results for diagnostic assay combinations and the agreement between diagnostic techniques. Considering UCP-LF CAA
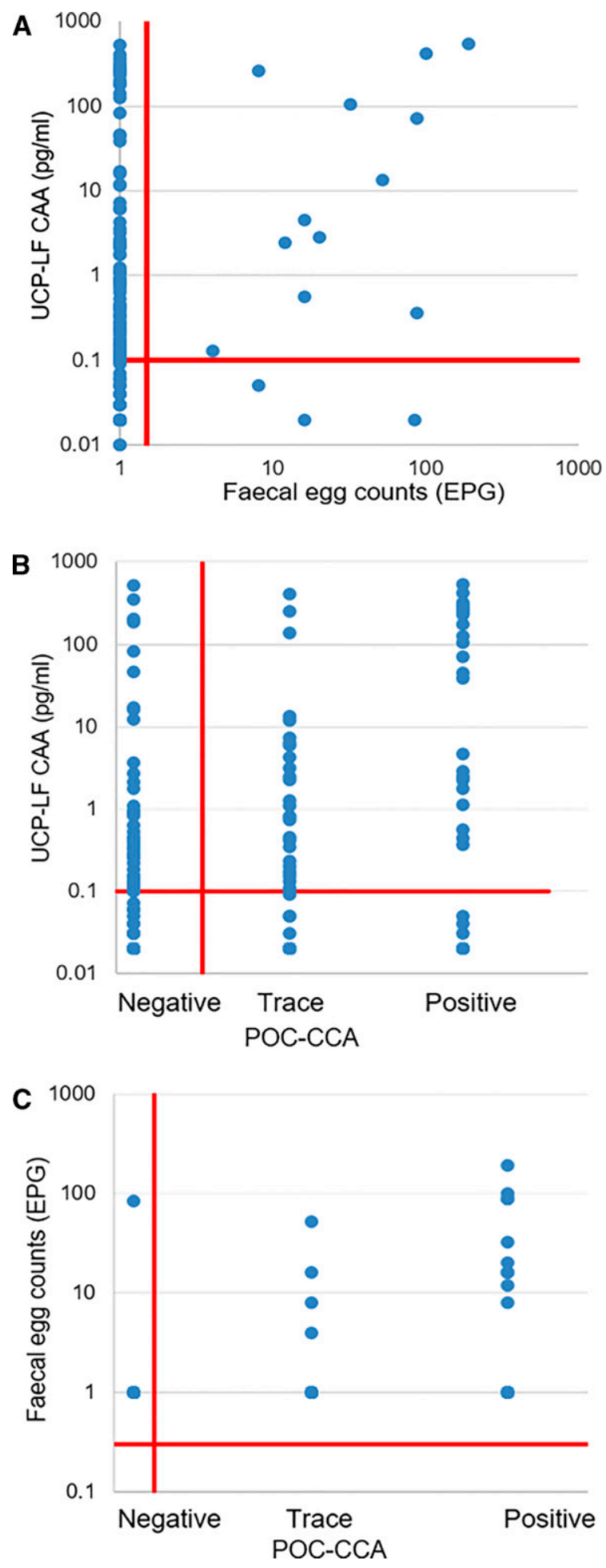

FIGURE 2. Correlation of Schistosoma mansoni urine circulating anodic antigen (CAA) levels and fecal egg counts (EPG) or point-ofcare (POC)-CCA intensity scores. (A) Correlation of CAA levels $(\mathrm{pg} / \mathrm{mL})$ determined by UCAA2000 and fecal egg counts (mean EPG of at least three slides). (B) Correlation of CAA levels $(\mathrm{pg} / \mathrm{mL}$ ) and POC-CCA intensity scores. (C) Correlation of fecal egg counts and POC-CCA.

indecisive and POC-CCA trace results as negative, a fair agreement between these two assays was found $(\kappa=0.24$, $P<0.001)$. The agreement between POC-CCA and six Kato-Katz thick smears was also fair $(\kappa=0.32, P<0.001)$. 
TABLE 3

Agreement between the different diagnostic methods

\begin{tabular}{lcccc}
\hline \multicolumn{5}{c}{ UCP-LF CAA } \\
\hline Kato-Katz $(6 \times)$ & Positive & Negative & Total & Kappa \\
Positive & 12 & 3 & 15 & \\
Negative & 76 & 287 & 363 & \\
Total & 88 & 290 & 378 & 0.18 \\
\hline \multicolumn{5}{c}{ POC-CCA } \\
\hline Kato-Katz (6×) & Positive & Negative & Total & Kappa \\
Positive & 10 & 5 & 15 & \\
Negative & 31 & 332 & 363 & 0.32 \\
Total & 41 & 337 & 378 & \\
\hline \multicolumn{5}{c}{ UCP-LF CAA } \\
POC-CCA & Positive & Negative & Total & Kappa \\
Positive & 23 & 65 & 88 & \\
Negative & 18 & 272 & 290 & 0.24 \\
Total & 41 & 337 & 378 \\
\hline
\end{tabular}

CCA = circulating cathodic antigen; POC = point-of-care; UCP-LF CAA = up-converting phosphor lateral flow circulating anodic antigen. Trace POC-CCA and indecisive UCP-LF CAA considered negative.

The agreement between UCP-LF CAA test and six Kato-Katz thick smears was poor $(\kappa=0.18, P<0.001)$.

\section{DISCUSSION}

Preventive chemotherapy with praziquantel is the current mainstay of schistosomiasis control. This strategy controls morbidity and might impact on the force of transmission, thus also playing a role in disease elimination in specific settings. ${ }^{6,7,31}$ In epidemiologic surveys, the diagnosis of schistosomiasis is usually based on stool and urine microscopy, which is inappropriate in low endemicity settings. Indeed, copromicroscopic approaches fail to detect light-intensity infections that are particularly common after several rounds of MDA with praziquantel. ${ }^{13}$ Hence, there is a need for new diagnostic tools with high specificity and sensitivity in low-endemicity settings.

The current study assessed the accuracy of POC-CCA and UCP-LF CAA for the diagnosis of S. mansoni among 9- to 12-year-old children in eight schools in the western part of Côte d'Ivoire that remained endemic for $S$. mansoni despite 5 years of preventive chemotherapy. ${ }^{15,32}$ The two urine-based assays detect pathogen-derived antigens. Similar to the detection of Schistosoma eggs, circulating antigens indicate an ongoing active infection, with the concentration of antigen directly linked to the Schistosoma worm burden. The overall S. mansoni prevalence was lower than $5 \%$ by six Kato-Katz thick smears derived from two consecutive stool samples. Urine samples assayed by the UCP-LF CAA test demonstrated a prevalence of $25.4 \%$, or $23.3 \%$ when indecisive results were considered negative. On the basis of the POC-CCA urine cassette test results, the S. mansoni prevalence was $30.7 \%$, or $10.9 \%$ when trace results were considered negative. The sensitivity of the UCP-LF CAA test was $80.7 \%$, whereas the corresponding sensitivity of POC-CCA and Kato-Katz assays were much lower; $37.6 \%$ and $13.8 \%$, respectively. In two children from different schools, $S$. haematobium eggs were detected with no indication of cross-reactivity with circulating antigens for S. mansoni.

We found that the two urine circulating antigen tests (UCP-LF CAA and POC-CCA) revealed several-fold higher S. mansoni prevalence than stool microscopy with multiple Kato-Katz thick smears. Indeed, when indecisive results were considered positive, the UCP-LF CAA test revealed a 6.4-fold higher prevalence of S. mansoni compared with Kato-Katz. Considering indecisive results as negative, there was still a 5.8-fold higher prevalence estimate. Interestingly, in the Gregbeu school where no S. mansoni positive case was observed by Kato-Katz, the prevalence measured by UCP-LF CAA assay was still above 10\%, regardless of whether indecisive results were included. The POC-CCA test revealed a prevalence of $23.4 \%$. Our observations confirm that circulating antigen tests have a much higher sensitivity for S. mansoni diagnosis than stool microscopy, particularly in low-endemicity settings. Our findings corroborate results from rural parts of northeastern Brazil and five ecological zones in Burundi. ${ }^{17,33}$ Moreover, our observations are in line with results of an analysis of banked urine samples from Cambodia and the Philippines for detection of S. mekongi and S. japonicum, respectively. ${ }^{34}$ In addition, a recent study comparing novel and standard diagnostic techniques for $S$. mekongi infection in Lao People's Democratic Republic and Cambodia reported that the UCP-LF CAA (UCAA2000 format) detected more S. mekongi cases than POC-CCA and multiple KatoKatz thick smears. ${ }^{19}$

Our results also confirmed that POC-CCA urine cassette test is more sensitive than multiple Kato-Katz thick smears, yet the sensitivities were low against our composite "gold" standard (37.6\% and $13.8 \%$, respectively). ${ }^{14,35}$ A recent study conducted in a village in Kafr El Dewar province in Egypt revealed that the sensitivity of POC-CCA was significantly higher than that of a PCR technique for S. mansoni diagnosis. $^{36}$ The main strengths of the POC-CCA urine cassette test are the relative low cost, simplicity of use, and the short duration until results are available compared with UCP-LF CAA test, which still requires a basic laboratory setup. The POC-CCA has been recommended for mapping schistosomiasis mansoni at large scale. ${ }^{37}$

Since our study, the format of the POC-CCA has been changed, and some issues with batch-to-batch variation have been reported. ${ }^{38}$ This mainly applies to the very low prevalence areas in $\mathrm{Brazil}^{39}$; in low-to-moderate areas, the POC-CCA provides a more accurate estimate of the true prevalence of $S$. mansoni compared with Kato-Katz thick smear readings. ${ }^{40}$ Caution must always be taken with the use of different production batches of rapid diagnostic tests, comparing the performance of consecutive batches using a set of reference samples.

We found that the combination of UCP-LF CAA and POCCCA showed high sensitivity for S. mansoni diagnosis; indeed, the sensitivity was close to $100 \%$. UCP-LF CAA indecisive and POC-CCA trace results are shortcomings when using circulating antigen assays. In case of the UCP-LF CAA test, indecisive samples in principle can be retested using a larger volume to get a definite result. Clearly, when pushing LF-based assays for highest sensitivity, trace results will always be an issue, especially with visual scored tests as the POC-CCA. Readerassisted tests like the UCP-LF CAA can omit trace (indecisive) results by setting a proper positivity threshold. In this study, data were analyzed considering trace (indecisive) results either as positive or negative.

\section{CONCLUSION}

Accurate diagnostic tools are pivotal for schistosomiasis risk profiling, measuring progress of gaining and sustaining control, and verification of elimination. Our research contributes to further evaluation of the accuracy of UCP-LF CAA for 
S. mansoni diagnosis. We found that UCP-LF CAA test (i.e., UCAA2000 format) is more sensitive than POC-CCA and Kato-Katz thick smears. Hence, the UCAA2000 format is a promising tool for diagnosis, particularly in low-endemicity settings. Compared with the POC-CCA test, the number of indecisive (trace) results are much fewer. However, the CAAbased test remains a laboratory-dependent tool and is not commercially available in low- and middle-income countries, where schistosomiasis is a public health problem and continues to impair the social and economic development. It is recommended to focus further on improving its affordability and field-friendly (POC) applicability.

Received January 9, 2021. Accepted for publication April 25, 2021.

Published online July 19, 2021.

Acknowledgments: We are grateful to the Schistosomiasis Consortium for Operational Research and Evaluation (SCORE) secretariat for reviewing the study protocol and for useful comments on an earlier version of the manuscript. We thank Claudia J. de Dood from the Near Patient Diagnostics research group at the Department of Cell and Chemical Biology from Leiden University Medical Center for the production of the UCP-LF CAA test and analysis of the urine samples with UCAA2000 format. We acknowledge assistance of Vincent A. E. Anzara, Diabaté Salia, Sadikou Touré, and Raphael G. Diabré with the laboratory work. We thank the team of the Laboratoire de Biologie et Santé at the Université Félix Houphouët-Boigny for all their efforts. We thank the staff of the Centre Suisse de Recherches Scientifiques en Côte d'Ivoire for administrative support. We are indebted to Dr. Aboulaye Meïté, Director of the Programme National de Lutte contre les Maladies Tropicales Negligées à Chimiothérapie Préventive at the Ministry of Health and his team, who treated children with praziquantel and provided logistic support. Teachers, parents, guardians, and children are acknowledged for their enthusiastic participation in the study. We are grateful to the health, education, and village authorities of Guemon, Cavally, and Haut-Sassandra for their contributions.

Financial support: This study was financially supported by the Rudolf Geigy Foundation for a 1-year postdoctoral fellowship granted to Rufin K. Assaré. The Schistosomiasis Consortium for Operational Research and Evaluation provided the point-of-care circulating cathodic antigen urine cassette tests and financially supported UCP-LF CAA tests.

Authors' addresses: Rufin K. Assaré, Unité de Formation et de Recherche Biosciences, Université Félix Houphouët-Boigny, Abidjan, Côte d'Ivoire, Groupe de Recherche Environnement et Santé, Centre Suisse de Recherches Scientifiques en Côte d'Ivoire, Swiss Tropical and Public Health Institute, Basel, Switzerland, and University of Basel, Basel, Switzerland, E-mail: hrufinass@yahoo.fr. Mathieu I. Tra-Bi, Unité de Formation et de Recherche Biosciences, Université Félix Houphouët-Boigny, Abidjan, Côte d'Ivoire, E-mail: trabimat@gmail. com. Jean T. Coulibaly, Unité de Formation et Recherche Biosciences, Université Félix Houphouët-Boigny, Abidjan, Côte d'Ivoire, Groupe de Recherche Environnement et Santé, Centre Suisse de Recherches Scientifiques en Côte d'Ivoire, Abidjan, Côte d'Ivoire, Swiss Tropical and Public Health Institute, Basel, Switzerland, and University of Basel, Basel, Switzerland, E-mail: couljeanvae@yahoo.fr. Mamadou Ouattara, Unité de Formation et de Recherche Biosciences, Université Félix Houphouët-Boigny, Abidjan, Côte d'Ivoire, Abidjan, Côte d'Ivoire and Groupe de Recherche Environnement et Santé, Centre Suisse de Recherches Scientifiques en Côte d'Ivoire, Abidjan, Côte d'Ivoire, E-mail: mamadou_ouatt@yahoo.fr. Paul L. A. M. Corstjens, Department of Cell and Chemical Biology, Leiden University Medical Center, Leiden, The Netherlands, E-mail: p.l.a.m.corstjens@lumc.nl. Eveline Hürlimann, Swiss Tropical and Public Health Institute, Basel, Switzerland, and University of Basel, Basel, Switzerland, E-mail: eveline.huerlimann@swisstph.ch. Govert J. van Dam, Department of Parasitology, Leiden University Medical Centre, Leiden, The Netherlands, E-mail: g.j.van_dam@lumc.nl. Jürg Utzinger, Swiss Tropical and Public Health Institute, and University of Basel, Basel, Switzerland, E-mail: juerg.utzinger@swisstph.ch. Eliézer K. N'Goran, Unité de Formation et de Recherche Biosciences, Université Félix
Houphouët-Boigny, Abidjan, Côte d'Ivoire, E-mail: eliezerngoran@ yahoo.fr.

This is an open-access article distributed under the terms of the Creative Commons Attribution (CC-BY) License, which permits unrestricted use, distribution, and reproduction in any medium, provided the original author and source are credited.

\section{REFERENCES}

1. McManus DP, Dunne DW, Sacko M, Utzinger J, Vennervald BJ, Zhou XN, 2018. Schistosomiasis. Nat Rev Dis Primers 4: 13.

2. Colley DG, Bustinduy AL, Secor E, King CH, 2014. Human schistosomiasis. Lancet 383: 2253-2264.

3. GBD 2017 DALYs and HALE Collaborators, 2018. Global, regional, and national disability-adjusted life-years (DALYs) for 359 diseases and injuries and healthy life expectancy (HALE) for 195 countries and territories, 1990-2017: a systematic analysis for the Global Burden of Disease Study 2017. Lancet 392: 1859-1922.

4. Fenwick $A, 2006$. Waterborne infectious diseases-could they be consigned to history? Science 313: 1077-1081.

5. World Health Organization, 2017. Schistosomiasis and soiltransmitted helminthiases: number of people treated in 2016. Wkly Epidemiol Rec 92: 749-760.

6. Lai YS et al., 2015. Spatial distribution of schistosomiasis and treatment needs in sub-Saharan Africa: a systematic review and geostatistical analysis. Lancet Infect Dis 15: 927-940.

7. King CH et al., 2020. Impact of different mass drug administration strategies for gaining and sustaining control of Schistosoma mansoni and Schistosoma haematobium infection in Africa. Am J Trop Med Hyg 103 (Suppl): 14-23.

8. WHO, 2013. Schistosomiasis: Progress Report 2001-2011, Strategic Plan 2012-2020. Geneva, Switzerland: World Health Organization.

9. Lamberton PH, Kabatereine NB, Oguttu DW, Fenwick A, Webster JP, 2014. Sensitivity and specificity of multiple Kato-Katz thick smears and a circulating cathodic antigen test for Schistosoma mansoni diagnosis pre- and post-repeated-praziquantel treatment. PLoS Negl Trop Dis 8: e3139.

10. Booth M, Vounatsou P, N'Goran EK, Tanner M, Utzinger J, 2003. The influence of sampling effort and the performance of the Kato-Katz technique in diagnosing Schistosoma mansoni and hookworm co-infections in rural Côte d'lvoire. Parasitology 127: 525-531.

11. Sandoval N, Siles-Lucas M, Perez-Arellano JL, Carranza C, Puente S, Lopez-Aban J, Muro A, 2006. A new PCR-based approach for the specific amplification of DNA from different Schistosoma species applicable to human urine samples. Parasitology 133: 581-587.

12. Al-Shehri $H$, Koukounari A, Stanton MC, Adriko M, Arinaitwe $M$, Atuhaire A, Kabatereine NB, Stothard JR, 2018. Surveillance of intestinal schistosomiasis during control: a comparison of four diagnostic tests across five Ugandan primary schools in the Lake Albert region. Parasitology 145: 1715-1722.

13. Utzinger J, Becker SL, van Lieshout L, van Dam GJ, Knopp S, 2015. New diagnostic tools in schistosomiasis. Clin Microbiol Infect 21: 529-542.

14. Colley DG et al., 2013. A five-country evaluation of a point-of-care circulating cathodic antigen urine assay for the prevalence of Schistosoma mansoni. Am J Trop Med Hyg 88: 426-432.

15. Assaré RK, Tra MBI, Ouattara M, Hürlimann E, Coulibaly JT, N'Goran EK, Utzinger J, 2018. Sensitivity of the point-of-care circulating cathodic antigen urine cassette test for diagnosis of Schistosoma mansoni in low-endemicity settings in Côte d'Ivoire. Am J Trop Med Hyg 99: 1567-1572.

16. Corstjens PLAM, van Lieshout L, Zuiderwijk M, Kornelis D, Tanke HJ, Deelder AM, van Dam GJ, 2008. Up-converting phosphor technology-based lateral flow assay for detection of Schistosoma circulating anodic antigen in serum. J Clin Microbiol 46: 171-176.

17. Sousa MS, van Dam GJ, Pinheiro MCC, Dood CJ, Peralta JM, Peralta RHS, Daher EF, Corstjens PLAM, Bezerra FSM, 2019. Performance of an ultra-sensitive assay targeting the circulating 
anodic antigen (CAA) for detection of Schistosoma mansoni infection in a low endemic area in Brazil. Front Immunol 10: 682.

18. Corstjens $P$ et al., 2020. Circulating anodic antigen (CAA): a highly sensitive diagnostic biomarker to detect active Schistosoma infections-improvement and use during SCORE. Am J Trop Med Hyg 103 (Suppl): 50-57.

19. Vonghachack $Y$ et al., 2017. Comparison of novel and standard diagnostic tools for the detection of Schistosoma mekongi infection in Lao People's Democratic Republic and Cambodia. Infect Dis Poverty 6: 127.

20. van Dam GJ et al., 2015. An ultra-sensitive assay targeting the circulating anodic antigen for the diagnosis of Schistosoma japonicum in a low-endemic area, People's Republic of China. Acta Trop 141: 190-197.

21. Corstjens PLAM, Nyakundi RK, de Dood CJ, Kariuki TM, Ochola EA, Karanja DMS, Mwinzi PNM, van Dam GJ, 2015. Improved sensitivity of the urine CAA lateral-flow assay for diagnosing active Schistosoma infections by using larger sample volumes. Parasit Vectors 8: 241

22. Corstjens PLAM, Hoekstra PT, de Dood CJ, van Dam GJ, 2017. Utilizing the ultrasensitive Schistosoma up-converting phosphor lateral flow circulating anodic antigen (UCP-LF CAA) assay for sample pooling-strategies. Infect Dis Poverty 6: 155.

23. WHO, 2006. Preventive Chemotherapy in Human Helminthiasis: Coordinated Use of Anthelminthic Drugs in Control Interventions. Geneva, Switzerland: World Health Organization.

24. Yap P, Fürst T, Müller I, Kriemler S, Utzinger J, Steinmann P, 2012. Determining soil-transmitted helminth infection status and physical fitness of school-aged children. J Vis Exp 66: e3966.

25. WHO, 2002. Prevention and control of schistosomiasis and soiltransmitted helminthiasis: report of a WHO expert committee. WHO Tech Rep Ser 912: 1-57.

26. Coulibaly JT et al., 2011. Accuracy of urine circulating cathodic antigen (CCA) test for Schistosoma mansoni diagnosis in different settings of Côte d'Ivoire. PLoS Negl Trop Dis 5: e1384.

27. Corstjens PLAM et al., 2014. Tools for diagnosis, monitoring and screening of Schistosoma infections utilizing lateral-flow based assays and upconverting phosphor labels. Parasitology 141: 1841-1855.

28. Knopp S et al., 2015. Sensitivity and specificity of a urine circulating anodic antigen test for the diagnosis of Schistosoma haematobium in low endemic settings. PLoS Negl Trop Dis 9: e0003752.
29. Alonzo TA, Pepe MS, 1999. Using a combination of reference tests to assess the accuracy of a new diagnostic test. Stat Med 18: 2987-3003.

30. Landis JR, Koch GG, 1977. The measurement of observer agreement for categorical data. Biometrics 33: 159-174.

31. Knopp S et al., 2019. Evaluation of integrated interventions layered on mass drug administration for urogenital schistosomiasis elimination: a cluster-randomised trial. Lancet Glob Health 7: e1118-e1129.

32. Ouattara M et al., 2021. Effectiveness of school-based preventive chemotherapy strategies for sustaining the control of schistosomiasis in Côte d'Ivoire: results of a 5-year cluster randomized trial. PLoS Negl Trop Dis 15: e0008845.

33. Clements MN et al., 2018. Latent class analysis to evaluate performance of point-of-care CCA for low-intensity Schistosoma mansoni infections in Burundi. Parasit Vectors 11: 111.

34. van Dam GJ, Odermatt P, Acosta L, Bergquist R, de Dood CJ, Kornelis D, Muth S, Utzinger J, Corstjens PL, 2015. Evaluation of banked urine samples for the detection of circulating anodic and cathodic antigens in Schistosoma mekongi and S. japonicum infections: a proof-of-concept study. Acta Trop 141: 198-203.

35. Bärenbold $O$ et al., 2018. Translating preventive chemotherapy prevalence thresholds for Schistosoma mansoni from the Kato-Katz technique into the point-of-care circulating cathodic antigen diagnostic test. PLoS Negl Trop Dis 12: e0006941.

36. Diab RG, Mady RF, Tolba MM, Ghazala RA, 2019. Urinary circulating DNA and circulating antigen for diagnosis of schistosomiasis mansoni: a field study. Trop Med Int Health 24: 371-378.

37. Colley DG et al., 2020. Contributions of the Schistosomiasis Consortium for Operational Research and Evaluation (SCORE) to schistosomiasis control and elimination: key findings and messages for future goals, thresholds, and operational research. Am J Trop Med Hyg 103 (Suppl): 125-134.

38. Viana AG et al., 2019. Discrepancy between batches and impact on the sensitivity of point-of-care circulating cathodic antigen tests for Schistosoma mansoni infection. Acta Trop 197: 105049.

39. Graeff-Teixeira $C$ et al., 2021. Low specificity of point-of-care circulating cathodic antigen (POCCCA) diagnostic test in a nonendemic area for schistosomiasis mansoni in Brazil. Acta Trop 217: 105863.

40. Colley DG et al., 2020. Evaluation, validation, and recognition of the point-of-care circulating cathodic antigen, urine-based assay for mapping Schistosoma mansoni infections. Am J Trop Med Hyg 103 (Suppl): 42-49. 